\title{
The determination of perceived tridimensional orientation by minimum criteria'
}

\author{
FRED ATTNEAVE ${ }^{2}$ AND ROBERT FROST \\ UNIVERSITY OF OREGON
}

The hypothesis investigated is that the perceived tridimensional orientation of an object is determined, in monocular vision, by tendencies to make the perceived object as simple as possible. Line drawings seen as "boxes" were viewed by Os who judged the slant (angle with frontal plane) of various edges. For every such edge, there is a determinate hypothetical slant consistent with perfect homogeneity of values on one or more of three variables (angle, length, and slope). Perceived slant was highly predictable from hypothetical slant, though always with some regression to the frontal plane. Results add support to a Prägnanz or minimum-principle theory of space perception.

The monocular perception of a three-dimensional space is commonly accounted for in terms of five or six "cues" to depth (listed in nearly every standard text), most of which go back to observations of Leonardo da Vinci. Gibson $(1950,1959)$.has vigorously opposed this type of explanation, with its implication of "unconscious inference," and proposes instead that tridimensional perception is directly controlled by certain higher-order stimulus variables such as gradients. A third alternative, first advanced by the classical Gestalt psychologists, is that the organization of tridimensional space follows the "Law of Pragnanz": that "psychological organization will always be as 'good' as the prevailing conditions allow [Koffka, 1935, pp. 110, 151, 159-164]." Hochberg (1964) has stated this idea more clearly and objectively as the "minimum principle": "that our nervous systems organize the perceived world in whatever way will keep changes and differences to a minimum." He proceeds to show how the traditional monocular "cues," as well as Gibson's stimulus-gradients, may be reinterpreted in this manner, as "cases of organizational simplicity." Earlier, Hochberg and McAlister (1953) hypothesized that "the less the amount of information needed to define a given organization as compared to the other alternatives, the more likely that the figure will be so perceived." Similarly, Attneave (1954) suggested that "the transformation of a figure to an aspect in which similarities among parts are maximized may be interpreted as the initial step in an efficient information-digesting process."

What remains to be demonstrated is that any strict functional relationship exists between the values of depth relationships actually perceived-e.g., the perceived slants of surfaces or edges-and the values that a principle of Prägnanz or minimum-complexity might demand. If we determine by geometry the tridimensional orientation that a pictured object would have to have to be as simple as possible by certain criteria, how accurately will the calculated orientation predict that which is perceived? This is the question investigated in the present research.

Stimuli

\section{METHOD}

The plan of the experiment may best be understood in terms of the stimulus variations employed.

Assume that Fig. 1 is an orthogonal projection of a parallelopiped. Any one of an infinite number of tridimensional

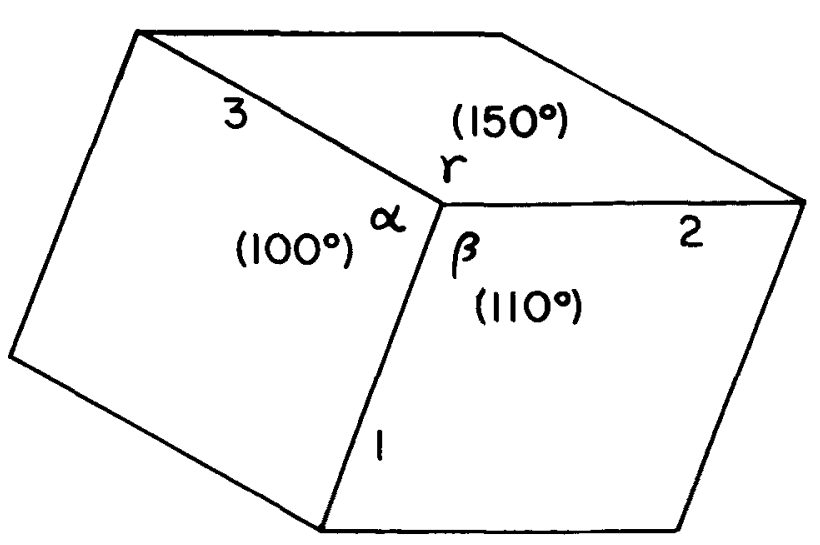

Fig. 1. Orthogonal projection of a parallelopiped.

objects could cast this projection; a parallelopiped is merely the simplest in the respect that all its angles are equal and right angles. Given this assumption, together with the angles $\alpha, \beta$, and $\gamma$ in the picture plane, we can calculate the angle $\phi$ that Edge 1 makes with the picture plane by the equation

$$
\Phi_{1}=\sin ^{-1} \sqrt{\cot \alpha \cot \beta} .
$$

For each of the other two edges, the slant is likewise calculated from the two plane angles bounded by its projection (see Appendix). Thus, for the particular values of Fig. 1:

$$
\begin{aligned}
\phi_{1} & =\sin ^{-1} \sqrt{\cot 100 \cot 110}=15 \mathrm{deg} \\
\phi_{2} & =\sin ^{-1} \sqrt{\cot 110 \cot 150}=51 \mathrm{deg} \\
\text { and } \phi_{3} & =\sin ^{-1} \sqrt{\cot 150 \cot 100}=35 \mathrm{deg} ;
\end{aligned}
$$

in other words, the three edges of the assumed box slant away from the $O$ at angles of 15,51 , and $35 \mathrm{deg}$. These values are of course unaffected by rotation of the figure in the picture plane.

Three different stimulus conditions are ilustrated in Fig. 2. All three boxes project the same angles $\alpha, \beta$, and $\gamma$, and hence have the same tridimensional orientation by the criterion just described.

In Condition 1, all the lines representing edges are of equal length in the picture plane; the obvious corrollary is that edges of the corresponding parallelopiped can not be equal. Moreover, "opposite" edges are represented by lines equal in slope (i.e., parallel) in the picture plane; this is equivalent to saying that the projection is an orthogonal one, or that "linear perspective" is not explicitly employed. Let us now hypothesize that the perceptual system seeks to minimize variability or diversity (or to maximize regularity, or homogeneity, or equality) of angles, lengths, and slopes, within the set of permissible tridimensional interpretations of the projection. If this is so, Condition 1 


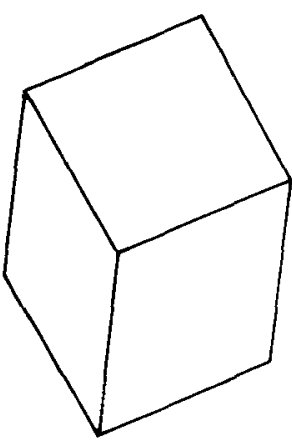

CONDITION I



CONOITION 2

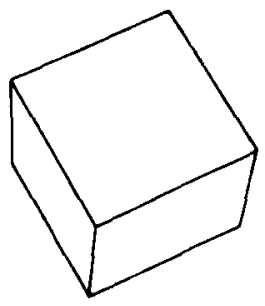

Fig. 2. Illustration of the three conditions. Hypothetically, all three parallelopipeds are in the same tridimensional orientation. presents the system with conflicting criteria: the angle criterion dictates the perceived orientation given by the inverse sine equations, but length and slope should tend to hold the figure flat, since both are maximally uniform in the frontal plane already, and become less so in any tridimensional arrangement.

In Condition 2, lengths of nonparallel lines are unequal in the picture plane. They become equal in exactly the same tridimensional orientation that equalizes angles; in other words, this is an orthogonal projection of a cube. (The new lengths are obtained simply by multiplying each of the old ones by $\cos \Phi$, the cosine of its slant as given by the inverse sine equation.) However, lines representing opposite edges remain parallel in the picture plane; therefore, the slope variable should still tend to hold the figure flat.

Finally, in Condition 3, "linear perspective" is added: i.e., slopes are equal, not in the picture plane, but in the same orientation that makes angles and lengths equal (See Appendix). This is to say that the picture is a polar projection of a cube, rather than an orthogonal projection as before. It provides the same retinal configuration as a real cube to an eye located at a particular viewing distance on a perpendicular passing through the central vertex of the figure. Perspective was calculated for the viewing distance used in the experiment, $47 \mathrm{~cm}$, but the reduced figure shown here should be viewed from about $20 \mathrm{~cm}$ for the perspective to be equivalent and correct.

To summarize: In Condition 1, angle should (according to the hypothesis) work for a particular, determinate depth impression; length and slope should work against it. In Condition 2, angle and length should work together for the same determinate orientation; slope should work to make the figure seem flat. In Condition 3 , all three variables should work together to make the edges appear to slant at the calculated angles.

It should be understood that there is no way to eliminate completely or neutralize any one of these variables; the nearest we can come to doing so is to make it consistent with the bidimensional case. The possibility that binocular disparity may play such a negative role in all three conditions must be considered later.

For each condition, nine figures were drawn; these were matched across conditions as in Fig. 2. Over the nine "boxes," the choice of angles $\alpha, \beta$, and $\gamma$ was varied to produce a wide range of $\Phi$-values (from about $8 \mathrm{deg}$ to $79 \mathrm{deg}$ ) among the 27 different edges. Under Condition 1, all the lines representing edges were $5 \mathrm{~cm}$ long in the picture plane; under the other conditions they were foreshortened (by the operations described above) to be consistent with projections of a 5-cm cube.

The stimulus figures were "drawn" in white thread on black cardboard: for each line, the thread was pulled taut over the surface between pinholes located at the vertex points. Figures constructed by this technique are cleaner and more accurate than india-ink drawings, and require less labor as well.

\section{Apparatus}

A view of the apparatus from above is shown in Fig. 3. By the combination of a half-silvered mirror and a polaroid arrangement, two fields-one monocular, the other binocular-are brought together in the same apparent space. The monocular field (right eye only) contains the stimulus figure, with its central vertex on the median axis at a virtual distance of $47 \mathrm{~cm}$. The binocular field contains a stick or rod $(.25 \mathrm{~cm}$ in diam and $19.5 \mathrm{~cm}$ long) that may be rotated in depth, i.e., in plane perpendicular to the frontal plane. This plane of rotation can be set at any desired tilt by rotating the drum on which the stick is mounted, but in the presented experiment it was kept at a tilt of $45 \mathrm{deg}$. The stick pivots on one end; this fixed end is located on the median axis at the same virtual distance $(47 \mathrm{~cm})$ as the stimulus figure; therefore the stick appears to pivot on the central vertex of the figure. It slants toward the $O$ at an angle that he controls by turning an external wheel.

The stimulus figure (centered on a heavy cardboard disc that fits snugly into a circular window) is oriented by $E$ so that one of its "edges" lies in the plane of rotation of the stick (i.e., at a 45-deg tilt). The task of $O$ is to align the stick with that edge, i.e., to make the stick a collinear extension of the edge in apparent tridimensional space. (To get a very crude intuitive notion of the task, the reader might try sticking a pin into the central vertex of one of the figures shown at a slant so that it appears tridimensionally collinear with one edge of the box.) If $O$ 's judgment is to have validity, he must perceive the slant of the stick accurately: to this end, the stick was painted white with $1 / 8$-in. black stripes every inch to give a strong basis for retinal disparity.

The apparatus provides an unusually satisfactory medium for slant judgments. Introspectively, the task seems totally lacking in any "intellectual" component. The $O$ feels as if he were objectively lining up the stick with the edge, and offsetting the stick by only 5 or 10 deg produces an "immediate" impression of discontinuity.

\section{Observers}

Four male psychology students, three graduates and one advanced undergraduate, served as Os. They were paid for their participation. The same procedure was followed with every $O$ individually.

\section{Procedure}

On each of 4 days, $O$ judged all 81 slants (three edges by nine boxes by three conditions). Order was random except that the three edges of a box were judged consecutively.

At the start of each trial on Days 1 and 2, the stick was placed in the frontal plane, i.e., on the picture-plane extension of the edge to be judged. On Days 3 and 4, all trials were started with the stick at $90 \mathrm{deg}$, i.e., pointed directly at the midpoint between 


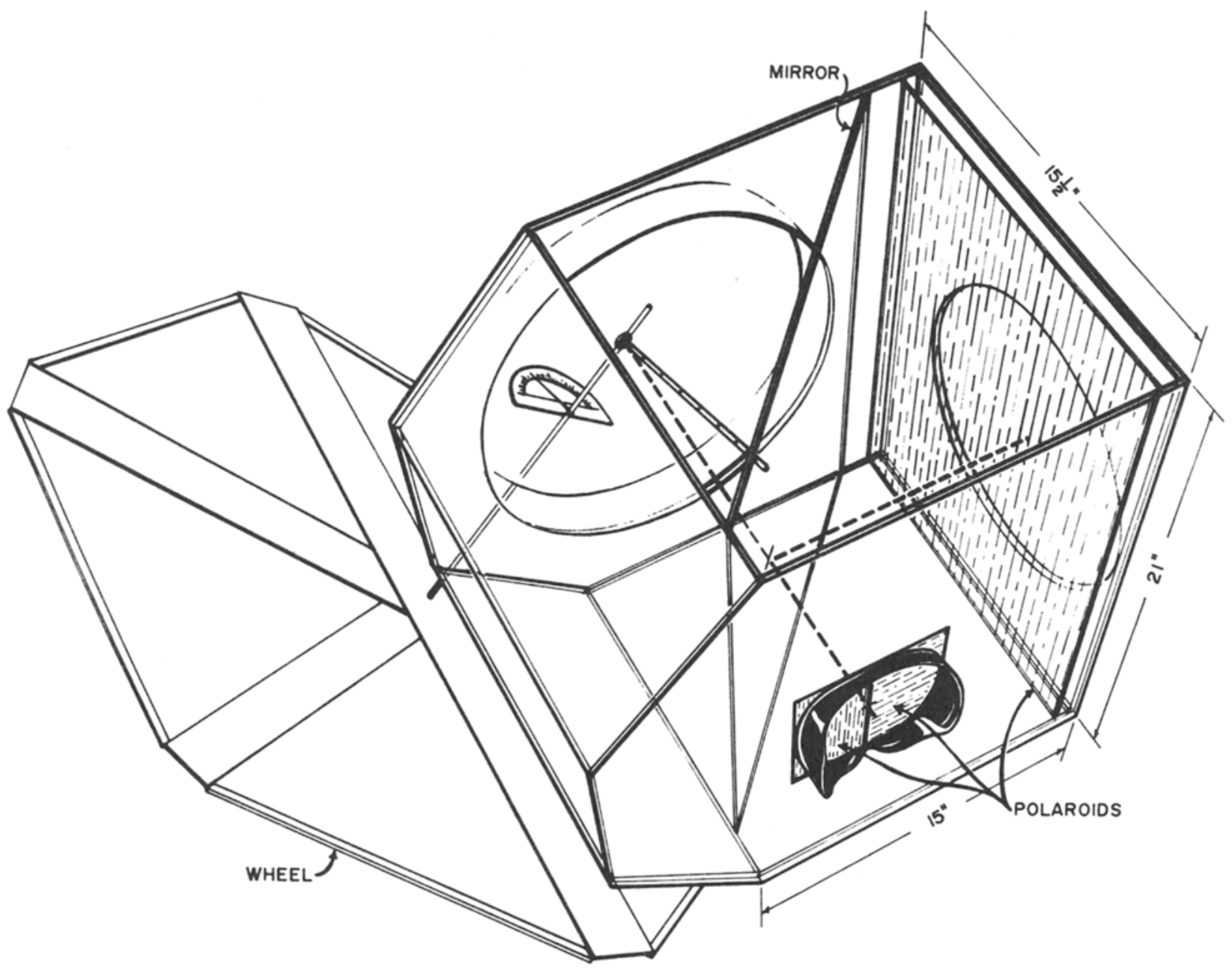

Fig. 3. The apparatus.

$O$ 's eyes. The $O$ was in no way prohibited from moving the stick back and forth in the region of his judgment, however.

As mentioned earlier, the plane of rotation of the stick was fixed in the present experiment at a 45-deg tilt (lower right to upper left). Within this restriction, the edge to be judged could still be placed in either of two orientations-in o'clock terms, at either $4: 30$ or $10: 30$. On Days 1 and 3 , the lower right orientation was used, on Days 2 and 4 , the upper left. This counterbalancing was considered advisable because of the slight left-right asymmetry of the perceived space, which was an unavoidable consequence of combining a monocular with a binocular field. (See the final paragraph of the Appendix.)

Each judgment was recorded as an angular deviation of the stick from the frontal plane. For each of the 81 edges, $O$ 's judgments were averaged over the 4 days, i.e., over the four combinations of the two procedural variables.

Instructions were simple and informal; all the Os accepted the alignment task as an easy and natural thing to do. Occasionally (for some Os more of ten than for others) the figure would reverse and appear concave in depth; on such occasions, $O$ was told merely to wait for it to reverse back into the box aspect and then make his alignment. The Os were well aware, intellectually, that the figures were physically flat drawings, and that they were viewing the figure with one eye and the stick with two. They were told nothing about the independent variables or basic design of the experiment, however.

\section{RESULTS}

The functional relationship between perceived and hypothetical slant is shown for each condition in Fig. 4. Even under Condition 1 , in which depth is entirely attributable to the angle variable, the relationship is a close one $(\mathrm{r}=.97)$, though the slope of the function is only .34. Addition of the length variable, in Condition 2, increases the slope to .59; with the further addition of the slope variable in Condition 3 , it increases a little more, to .63. This pattern is quite consistent over the four $O$ s; see Table 1.

The conflict inherent in Conditions 1 and 2 might be handled in either of two ways, depending upon how the perceptual system works: It might be resolved by some averaging process, or it might remain unresolved, and result in bimodal or otherwise unstable perceptions of slant. The data strongly support the former alternative. Judgments are almost as stable in Condition 1 as in Condition 3; the increase in correlation coefficients is attributable mainly to increase in range of judgments rather than to decrease in "error" variance. Moreover, judgments of a given edge do not in any condition show bimodality. 3 




Fig. 4a. Condition 1: Judged slant as a function of hypothetical slant. Angles are equalized at the hypothetical value; lengths and slopes are equal in the frontal plane.

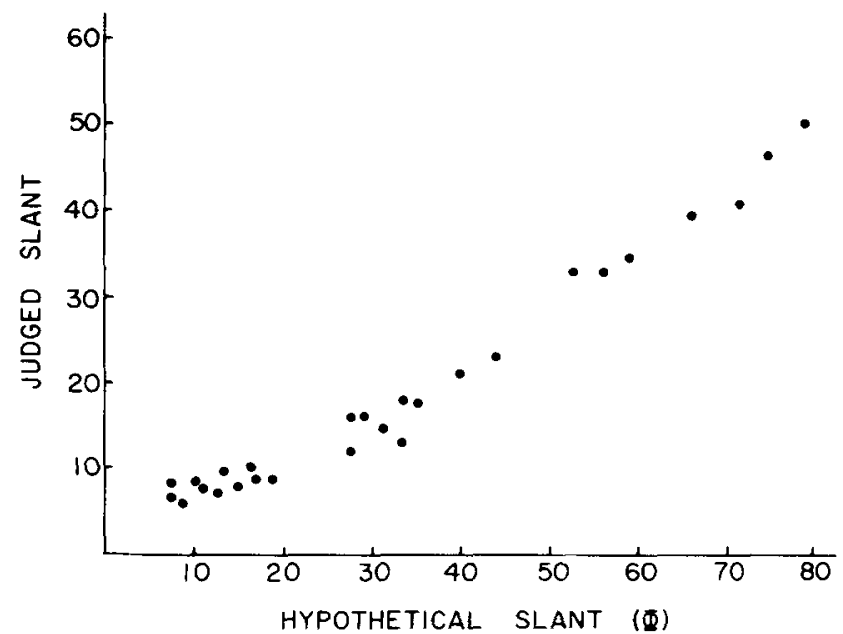

Fig. 4b. Condition 2: Judged slant as a function of hypothetical slant. Angles and lengths are equalized at the hypothetical value; slopes are equal in the frontal plane.



Fig. 4c. Condition 3: Judged slant as a function of hypothetical slant. Angles, lengths, and slopes are all equalized at the hypothetical value.
Table 1

Prediction of Judged Slant from Hypothetical Slant, by Observer and Condition

\begin{tabular}{|c|c|c|c|}
\hline Observer & Condition & $\begin{array}{l}\text { Correlation } \\
\text { Coefficient }\end{array}$ & Least-Squares Fit \\
\hline T.W. & $\begin{array}{l}1 \\
2 \\
3\end{array}$ & $\begin{array}{l}.92 \\
.96 \\
.97\end{array}$ & $\begin{array}{l}Y=.40 X+1.76 \\
Y=.69 X-6.87 \\
Y=.72 X-6.13\end{array}$ \\
\hline J.L. & $\begin{array}{l}1 \\
2 \\
3\end{array}$ & $\begin{array}{l}.94 \\
.98 \\
.99\end{array}$ & $\begin{array}{l}Y=.34 X+8.86 \\
Y=.55 X+3.70 \\
Y=.59 X+4.12\end{array}$ \\
\hline C.S. & $\begin{array}{l}1 \\
2 \\
3\end{array}$ & $\begin{array}{l}.96 \\
.98 \\
.97\end{array}$ & $\begin{array}{l}Y=.26 X+9.42 \\
Y=.53 X+2.15 \\
Y=.56 X+2.11\end{array}$ \\
\hline D.T. & $\begin{array}{l}1 \\
2 \\
3\end{array}$ & $\begin{array}{l}.95 \\
.96 \\
.98\end{array}$ & $\begin{array}{l}Y=.37 X+3.40 \\
Y=.59 X-0.98 \\
Y=.66 X-0.98\end{array}$ \\
\hline Pooled Data & $\begin{array}{l}1 \\
2 \\
3 \\
\end{array}$ & $\begin{array}{l}.97 \\
.98 \\
.99 \\
\end{array}$ & $\begin{array}{l}Y=.34 X+5.86 \\
Y=.59 X-0.49 \\
Y=.63 X-0.17\end{array}$ \\
\hline
\end{tabular}

The determinacy of the obtained relationships between perceived and hypothetical slant is highly impressive. That the relationship is not one of identity is quite understandable in Conditions 1 and 2 , since in these conditions we can specify either one or two variables that should (according to a minimum principle) tend to hold the perceived figure in the frontal plane. In Condition 3, however, no such conflict was supposed to be present; why, then, does the slope of the function (Fig. 4c) remain less than one? ${ }^{4}$ Unless we assume some irreducible principle of regression to the frontal plane, it seems likely that the $O$ is receiving some residual, unspecified information that is tending to flatten perceived slant. Stereopsis, or rather the lack thereof, is one plausible candidate for this role. It is fairly obvious that if the figure had been viewed binocularly, the lack of interocular disparity between contours would have made for an impression of flatness. We should like to be able to assume that in monocular vision the mechanisms of stereopsis simply suspend operation, and have no effect of any sort on perception, but this assumption may be unwarranted. The alternative possibility is that the system interprets an absence of contour disparity as evidence that the figure lies in the frontal plane, whether the lack of disparity results from monocular vision or from a binocular view of a figure that is in fact in the frontal plane. An attempt will be made in a future study to clarify this issue. ${ }^{5}$

Effects of linear perspective (perception of slant consistent with equalization of slopes) may be much more important generally than the small difference in results between Conditions 2 and 3 suggests. (See Freeman, 1966a, b, for example.) An experimental design that allowed us to assign ecologically valid weights to the variables on which minimizing occurs would have to be far more elaborate than the present one. One should bear in mind that linear perspective is peculiar to the object size and viewing distance employed. It is quite possible, moreover, that linear perspective would have a greater effect if it were introduced as the second, rather than the third, step of stimulus variation, i.e., with lengths still equal in the picture plane.

Throughout this account we have suggested that depth perception is determined by tendencies to minimize the variability of angles, lengths, and slopes. An alternative view of what it is that the system seeks to minimize deserves consideration, even if it appears less parsimonious as an explanation of the present results. Observations on ambiguous triangles recently reported by one of the authors (Attneave, 1968) support the belief that visual objects are perceived in terms of an internal Cartesian reference system. The hypothesis that the present figures are perceived in a way that simplifies not their 
internal relationships, but their relationships to such an underlying reference system, could explain the effects of both the angle and the slope variables: consider that a parallelopiped is a Cartesian object par excellence. Further assumptions would be required to incorporate the length variable into this sort of model. Experiments that we hope will decide between these altematives are under way.

In any case, the results of the present experiment seem clearly more consistent with some version of a Prägnanz theory than with a treatment in terms of either "cues" or "higher-order stimulus variables." (1) A cue theory, as we understand it, would have to assume the neural equivalent of a massive table listing correspondences between particular combinations of angles, for example, and particular slants. With all due allowance for approximation, interpolation, etc., this would require a formidable number of associations. Note further that different shapes would require different tables, and that the system would have to decide which table to use on the basis of the projection, without recourse to tridimensional information that the table has yet to provide. (2) We have, in fact, employed a "higher-order stimulus variable" -- the $\Phi$ that was calculated by the inverse sine equation-as a rather successful basis for predicting slant judgments. To suppose that the visual system likewise solves this equation to abstract such a variable strains one's credulity, the more so as one considers in detail the operations involved in the transformation. This type of explanation would be more plausible if it could be shown that a simpler transformation on the retinal data yielded better prediction of judgments. However, different shapes would still require different transformations, and the choice of transformation presents the same decision problems as choice of table in the previous account. (3) To either of these models it may be possible to append some explanation of the tendency to perceive things simply, but in neither case is any such tendency an integral part of the model. A Prägnanz principle assumes a teleological system (as Koffka, 1935, explicitly

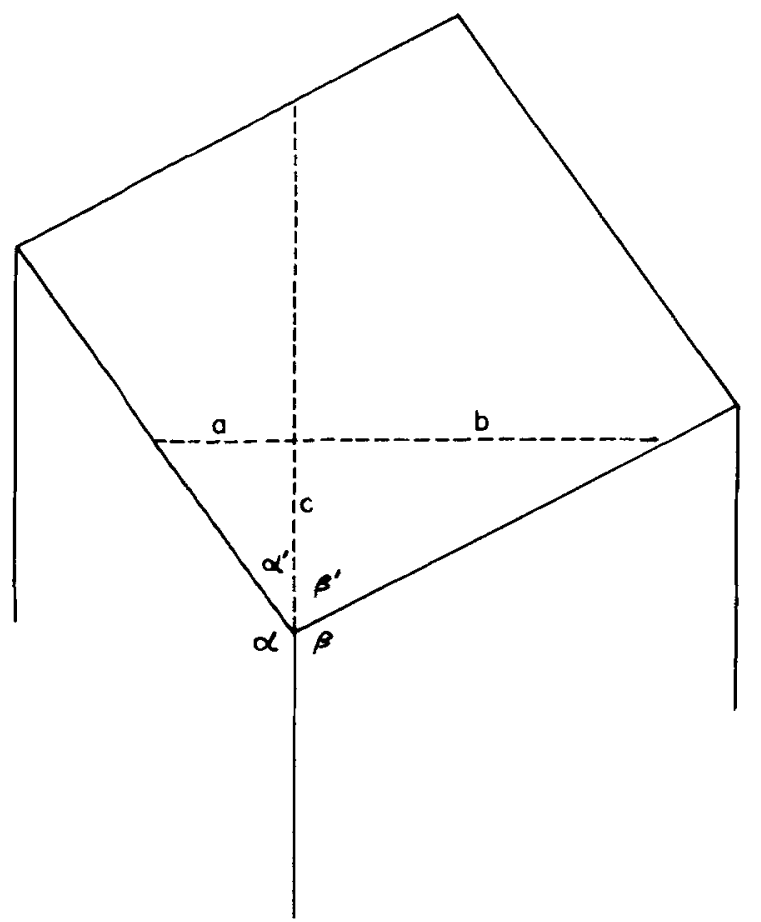

Fig. 5. Ilustrating calculation of tridimensional orientation from picture-plane angles. recognized) in which simplicity has the status of a final cause, or goal-state. It assumes that the rules of perspective (or some approximation thereto) are implicit in an analog medium representing physical space, within which the representation of an object moves toward a stable state characterized by "figural goodness" or minimum complexity. This type of theory has extraordinary advantages of generality and economy. It is quite inadequately specific, leaving much to be learned about the variables that the system tends to minimize, and how they combine.

\section{APPENDIX}

(1) Derivation of inverse sine equation. The orthogonal projection of a portion of a parallelopiped is shown in Fig. 5. The projection of the edge in which we shall be interested is oriented vertically, to simplify discussion. The extension of this line above the central intersection may be considered, tridimensionally, the locus of intersection of the median plane of the $O$ with the top of the box.

Through an arbitrary point on this extension, a perpendicular is drawn to the two edges, forming two right triangles with the common side, $c$. Note that these are right triangles, both on the surface of the box and in projection; also that Sides $a$ and $b$ are of the same length in the projection as on the surface of the solid. Segment $\mathrm{c}$ is foreshortened, however; therefore, we shall use the term $c_{2}$ to refer to its length in the picture plane and $c_{3}$ for its length on the box top. Likewise, $\alpha_{3}^{\prime}$ and $\beta_{3}^{\prime}$ will refer to the tridimensional values of those angles, and $\alpha_{2}^{\prime}$ and $\beta_{2}^{\prime}$ to their projections. Finally, let us define $\Theta$ as the angle between $c_{2}$ and $c_{3}$, i.e., the slant of the box top relative to the picture plane.

We can now write

$$
\begin{aligned}
& \text { and } \left.\quad \begin{array}{c}
\cot \alpha_{2}^{\prime}=\frac{c_{2}}{a} \\
\cot \beta_{2}^{\prime}=\frac{c_{2}}{b} .
\end{array}\right\} \\
& \text { Since } \\
& c_{2}=c_{3} \cos \Theta, \\
& \left.\begin{array}{l}
\cot \alpha_{2}^{\prime}=\frac{c_{3} \cos \Theta}{a}, \\
\cot \beta_{2}^{\prime}=\frac{c_{3} \cos \Theta}{b},
\end{array}\right\} \\
& \text { or, } \\
& \text { and } \\
& \frac{c_{3}}{\mathrm{a}}=\frac{\cot \alpha_{2}^{\prime}}{\cos \Theta}, \\
& \frac{c_{3}}{b}=\frac{\cot \beta_{2}^{\prime}}{\cos \Theta} \text {. } \\
& \left.\begin{array}{l}
\cot \alpha_{3}^{\prime}=\frac{c_{3}}{a}, \\
\cot \beta_{3}^{\prime}=\frac{c_{3}}{b} ;
\end{array}\right\}
\end{aligned}
$$

therefore

$$
\cot \alpha_{3}^{\prime}=\frac{\cot \alpha_{2}^{\prime}}{\cos \Theta}, \quad \mid
$$

and

$$
\cot \beta_{3}^{\prime}=\frac{\cot \beta_{2}^{\prime}}{\cos \Theta} \quad \int
$$

But since $\alpha_{3}^{\prime}+\beta_{3}^{\prime}=90 \mathrm{deg}$,

therefore

$$
\cot \alpha_{3}^{\prime} \cot \beta_{3}^{\prime}=1
$$

and

$$
\frac{\cot \alpha_{2}^{\prime} \cot \beta_{2}^{\prime}}{\cos ^{2} \Theta}=1 \text {, }
$$

$$
\cos \Theta=\sqrt{\cot \alpha_{2}^{\prime} \cot \beta_{2}^{\prime}} .
$$

By the Eq. 9 we can calculate the slant of any surface of the box, given the projected angles. The further step of specifying the slant, $\Phi$, of the perpendicular edge is a trivial one. Since $\Theta+\Phi=90 \mathrm{deg}$,

therefore

$$
\cos \Theta=\sin \Phi ;
$$






Fig. 6. Illustrating calculation of linear perspective.

or,

$$
\begin{gathered}
\sin \Phi=\sqrt{\cot \alpha_{2}^{\prime} \cot \beta_{2}^{\prime}}, \\
\Phi=\sin ^{-1} \sqrt{\cot \alpha_{2}^{\prime} \cot \beta_{2}^{\prime}} .
\end{gathered}
$$

Finally, we can substitute the more obvious obtuse angles $\alpha$ and $\beta$ for $\alpha_{2}^{\prime}$ and $\beta_{2}^{\prime}$ (since they have the same cotangents except for a change of sign that disppears in the multiplication) and write the equation as it appears in the text:

$$
\Phi=\sin ^{-1} \sqrt{\cot \alpha \cot \beta} .
$$

To the best of our knowledge, relationships like those just derived have not previously been considered in the study of monocular depth perception. The additional calculations required for Conditions 2 and 3 are more familiar, but will be described briefly.

(2) Foreshortening of lengths in orthogonal projection. Given the slant $\Phi$, the relationship between the tridimensional length of an edge and that of its projection is simply

$$
\ell_{2}=\ell_{3} \cos \Phi .
$$

(3) Linear perspective. A polar projection may be constructed from an orthogonal projection in the following manner. Assuming that the central vertex of the figure lies in the picture plane, and given the slants and lengths of the edges, the distance of each of the remaining six visible vertices behind the picture plane may easily be calculated. This calculation will not be detailed. In Fig. 6 , Point $V$ represents the central vertex, and Point $\mathrm{C}$ any one of the remaining corners; the plane of Fig. 6 is that which contains both points and is orthogonal to the frontal plane. The O's eye, $\mathrm{E}$, is located directly in front of $\mathrm{V}$ at a distance of $\mathrm{d}_{\mathrm{v}}$; $\mathrm{C}$ is located at a distance $d_{b}$ behind the picture plane. Point $P_{o}$ is the orthogonal projection of $C$ and $P_{p}$, the polar or perspective projection; $d_{o}$ and $d_{p}$ are their respective distances from $V$. Note that $C_{p} P_{p}$ and $E P_{p} V$ are similar triangles. It follows that

$$
\frac{d_{o}-d_{p}}{d_{b}}=\frac{d_{p}}{d_{v}}
$$

or,

$$
d_{p}=d_{o}\left(\frac{d_{v}}{d_{b}+d_{v}}\right)
$$

The parenthetical term in Eq. 16 is a shrinkage factor by which the distance from the central vertex to a given comer must be contracted in going from an orthogonal to a polar projection.

In the calculations for Condition 3 a Cyclopean point of view was assumed: i.e., Point E of Fig. 6 was located midway between O's eyes. Whether or not this was proper is debatable, involving the obscure question of what the visual system takes as "straight ahead" in a monocular field that is fused with a binocular field. The issue is somewhat academic, however, for two reasons: (a) the difference in linear perspective between Cyclopean and righteye viewpoints is fairly small (less than $4 \mathrm{deg}$, in terms of hypothetical slant); and (b) trials were counterbalanced for left-right asymmetry.

\section{REFERENCES}

ATTNEAVE, F. Some informational aspects of visual perception. Psychological Review, 1954, 61, 183-193.

ATTNEAVE, F. Triangles as ambiguous figures. American Journal of Psychology, 1968, 18, 643-662.

FREEMAN, R. B. Function of cues in the perceptual learning of visual slant: An experimental and theoretical analysis. Psychological Monographs, 1966, No. 610 .

FREEMAN, R. B. Effect of size on visual slant. Journal of Experimental Psychology, 1966, 71, 96-103.

GIBSON, J. J. Penception of the visual world. Boston: Houghton Mifflin, 1950.

GIBSON, J. J. Perception as a function of stimulation. In S. Koch (Ed.), Psychology: A study of a science. Vol. 1. New York: McGraw-Hill, 1964.

HOCHBERG, J. E. Perception. Englewood Cliffs: Prentice-Hall, 1964.

HOCHBERG, J. E., \& BROOKS, V. The psychophysics of form: Reversible-perspective drawings of spatial objects. American Journal of Psychology, 1960, 73, 337-354.

HOCHBERG, J. E., \& MCALISTER, E. A quan titative approach to figural "goodness." Journal of Experimental Psychology, 1953, 46, 36 1-364.

KOFFKA, K. Principles of Gestalt psychology. New York: Harcourt Brace, 1935.

\section{NOTES}

1. This research was supported in part by the Air Force Office of Scientific Research, Grant 973-66, and in part by the Advanced Research Projects Agency of the Department of Defense, Contract F44620-67C-0099. Mr. Nick Garshnek deserves special credit for his contributions to the design of the apparatus, as well as for its physical construction.

2. Address: Department of Psychology, University of Oregon, Eugene, Oregon 97403.

3. The figures for which Hochberg and his associates $(1953,1960)$ found alternation of bidimensional and tridimensional perception are perhaps characterized by two distinct "valleys" of complexity, one in the picture plane, the other in depth. Our Ss behave as if the frontal plane exerted a "pull" on the figure, but never captured it. We considered the possibility that the strength of the "pull" might still be affected, within a condition, by incidental ditterences in trontal-plane complexity. In Condition 1, for example, the plane angles are more variable in some figures than in others, and one might expect the more homogeneous to show greater regression to the frontal plane. When residuals from the regression line for Condition 1 (Fig. 4a) are plotted against the range of the central angles $(\alpha, \beta$, and $\gamma)$ of the figures to which the values apply, the correlation is quite unimpressive: $r=.16$. There is a bare suggestion of a weak, nonlinear relationship in the fact that five of the six edges of the two most "homogeneous" figures (with angle ranges of $10 \mathrm{deg}$ and $20 \mathrm{deg}$ ) were judged closer to the frontal plane than the regression line predicted. It appears fairly likely (especially in view of the kinds of complexity measures that Hochberg et al found predictive) that the perceptual system performs a nonlinear or nonmetric evaluation of variability that may be approximated simply by counting the number of values that are different on the continuum in question. In this sense, our figures were all about equally variable in the frontal plane, within any given condition.

4. Impressions of slant from even the best of photographs show the same sort of compromise. The reader is invited to take a frontal view of some photograph like Fig. 35,61 , or 70 of Gibson's book (1950) and orient his hand parallel to the apparent plane of the pictured ground or floor. He will find that his judgment deviates rather markedly from the horizontal, toward the picture plane.

5. Whether or not stereoscopic depth perception can itself be subsumed under a minimum principle is an interesting question, but quite beyond the scope of the present article.

(Accepted for publication April 24, 1969.) 